\title{
Análise bibliométrica sobre parâmetros multidimensionais da voz associados à tireoidectomia nos periódicos brasileiros de Fonoaudiologia
}

\section{Bibliometric analysis on the voice multidimensional outcomes associated with thyroidectomy in the Brazilian journals of Speech, Language and Hearing Sciences}

\section{Análisis bibliométrico de los parámetros multidimensionales de la voz asociados a la tiroidectomía en revistas brasileñas de Logopedia}

\author{
Lília da Silva Lira* (iD) \\ Luciane Spinelli de Figueiredo Pessoa* (1) \\ Maria Fabiana Bonfim de Lima-Silva* \\ Leandro Pernambuco* (i)
}

\section{Resumo}

Objetivo: Analisar o perfil da produção científica publicada nos periódicos brasileiros de Fonoaudiologia sobre parâmetros multidimensionais da voz associados à tireoidectomia. Método: revisão bibliográfica com análise bibliométrica e busca realizada nas bases de dados dos quatro periódicos brasileiros especializados em Fonoaudiologia, indexados entre 2005 e 2019. Foram cruzados os descritores disfonia, tireoidectomia e neoplasias da glândula tireoide, sendo incluídos os artigos originais, de revisão, estudos de caso, comunicações breves e resenhas editoriais que apresentassem resultados sobre parâmetros

* Universidade Federal da Paraíba, João Pessoa, Paraíba, Brasil.

Contribuição dos autores:

LSL - contribuiu com a concepção, coleta, análise dos dados, escrita, análise crítica e revisão final do artigo. LSFP e MFBL-S - contribuíram com a análise crítica e revisão final do artigo. LP - contribuiu com a orientação, análise dos dados, escrita e revisão final do artigo.

E-mail para correspondência: Lília da Silva Lira - lilial.lira@gmail.com Recebido: $28 / 03 / 2020$

Aprovado: $28 / 5 / 2020$ 
perceptivoauditivos, acústicos e/ou de autoavaliação da voz antes ou após tireoidectomia. Foram extraídas a região e instituição em que o estudo foi realizado, a quantidade de instituições envolvidas, número de autores, grau acadêmico dos autores, periódico de publicação, tipo de artigo, desenho de estudo, tamanho da amostra e parâmetros vocais avaliados. Resultado: Foram incluídos cinco estudos: dois realizados na região Sudeste, dois no Nordeste e um no Sul; três com parceria interinstitucional; quatro em que o primeiro autor era fonoaudiólogo; coautores fonoaudiólogos e médicos com grau acadêmico desde graduação até doutorado; número de autores entre três e sete. Quatro estudos foram transversais e abordaram apenas autoavaliação vocal. Três estudos foram publicados como artigos originais, um como comunicação breve e um como resenha. O tamanho amostral oscilou entre 12 e 60 indivíduos; cada periódico publicou, no mínimo, um artigo sobre o tema. Conclusão: existe baixa produção científica publicada nos periódicos brasileiros de Fonoaudiologia sobre parâmetros multidimensionais da voz associados à tireoidectomia, já que em 15 anos, foram publicados apenas cinco estudos sobre o tema.

Palavras chave: Disfonia; Voz; Tireoidectomia; Fonoaudiologia; Bibliometria. Brasil.

\section{Abstract}

Objective: To analyze the profile of scientific production published in Brazilian Speech, Language and Hearing Sciences journals on multidimensional voice parameters associated with thyroidectomy. Method: Bibliographic review with bibliometric analysis and search made in the databases of four Brazilian journals specialized in Speech, Language and Hearing Sciences, indexed between 2005 and 2019.The descriptors of dysphonia, thyroidectomy and thyroid gland neoplasms were cross-referenced, including original articles, review articles, case studies, short communications and editorial reviews presenting results on perceptive-auditory, acoustic and/or self-evaluation parameters of the voice before or after thyroidectomy. We extracted the geographic region and institution where the study was carried out, the number of institutions involved, the number of authors, the authors' academic degree, the publication periodic, the type of article, the study design, the sample size and the vocal parameters evaluated. Results: Five studies were included: two carried out in the Southeast region, two in the Northeast and one in the South; three with inter-institutional partnership; four in which the first author was a speech therapist; speech therapists and physicians with academic degrees from undergraduate to $\mathrm{PhD}$; number of authors between three and seven. Four studies were transversal and approached only vocal self-evaluation. Three studies were published as original articles, one as a brief communication and one as a review. The sample size ranged from 12 to 60 individuals; each journal published at least one article on the subject. Conclusion: there is a low scientific production published in Speech, Language and Hearing Sciences journals on multidimensional voice parameters associated with thyroidectomy, since in 15 years, only five studies on the subject have been published.

Keywords: Dysphonia; Voice; Thyroidectomy; Speech, Language and Hearing Sciences; Bibliometrics. Brazil.

\section{Resumen}

Objetivo: Analizar el perfil de la producción científica publicada en las revistas brasileñas de Logopedía sobre los parámetros multidimensionales de la voz asociados a la tiroidectomía. Método: Revisión bibliográfica con análisis bibliométrico y búsqueda en las bases de datos de cuatro revistas brasileñas especializadas en Logopedia, indizadas entre 2005 y 2019. Se hicieron referencias cruzadas de los descriptores de disfonía, tiroidectomía y neoplasias de la glándula tiroides, incluidos artículos originales, artículos de revisión, estudios de casos, comunicaciones breves y revisiones editoriales que presentaban los resultados sobre parámetros perceptivos-auditivos, acústicos y/o de autoevaluación de la voz antes o después de la tiroidectomía. Se extrajo la región y la institución en la que se realizó el estudio, el número de instituciones involucradas, el número de autores, el grado académico de los autores, la revista de publicación, el tipo de artículo, el diseño del estudio, el tamaño de la muestra y los parámetros vocales evaluados. Resultados: Se incluyeron cinco estudios: dos realizados en la región Sudeste, dos en el Noreste y uno en el Sur; tres con asociación interinstitucional; cuatro en los que el 
primer autor fue un logopeda; logopedas y médicos con títulos académicos desde la licenciatura hasta el doctorado; número de autores entre tres y siete. Cuatro estudios fueron transversales y abordaron sólo la autoevaluación vocal. Se publicaron tres estudios como artículos originales, uno como una comunicación breve y otro como una revisión. El tamaño de la muestra osciló entre 12 y 60 individuos; cada revista publicó al menos un artículo sobre el tema. Conclusión: existe una baja producción científica publicada en las revistas brasileñas de Logopedía sobre los parámetros multidimensionales de la voz asociados a la tiroidectomía, ya que en 15 años sólo se han publicado cinco estudios sobre el tema.

Palabras clave: Disfonía; Voz;Tireoidectomía; Fonoaudiología; Bibliometría. Brasil.

\section{Introdução}

A tireoide é uma glândula composta por dois lobos laterais e um istmo central, localizada na região do pescoço, anterior à cartilagem cricoide e traqueia e ligeiramente inferior à cartilagem tireoide. ${ }^{1}$ Por meio da atividade dos hormônios que produz, a tireoide é responsável pelo crescimento, desenvolvimento e regulação de processos metabólicos do organismo. ${ }^{2}$

A atividade tireoidiana pode ser afetada por doenças benignas e malignas e uma das opções de tratamento é a tireoidectomia, procedimento para remoção parcial ou total da glândula. ${ }^{3-6} \mathrm{~A}$ estreita proximidade anatômica da tireoide com os nervos laríngeos expõe o paciente submetido à tireoidectomia ao risco de alterações vocais após o procedimento $^{1,3-8}$. Essas alterações podem ocorrer devido a fatores como extensão da cirurgia ${ }^{1,8}$, técnica operatória utilizada ${ }^{9}$, intubação orotraqueal ${ }^{4,5}$ e manipulação dos nervos laríngeos ${ }^{3-5}$ com possibilidade de paresia ou paralisia de pregas vocais ${ }^{3,6}$.Além disso, existem pacientes com sintomas faringolaríngeos como rouquidão, cansaço ao falar, garganta seca, pigarro, prurido na garganta e dificuldades para alcançar frequências altas ${ }^{10,11}$ mesmo na ausência de lesões nervosas periféricas, condição que vem sendo denominada "síndrome pós-tireoidectomia"3e que pode levar os pacientes a buscar tratamento fonoaudiológico ${ }^{7}$.

Diante desse cenário, entende-se que existe uma demanda de investimento em produções científicas que abordem a associação entre tireoidectomia e suas possíveis sequelas nos parâmetros perceptivoauditivos, acústicos e de autoavaliação que são componentes multidimensionais da voz. No Brasil, as revistas especializadas em Fonoaudiologia se apresentam com grande potencial de alcance entre os fonoaudiólogos, promovendo acesso a evidências científicas que contribuem para a prática clínica. Nesse sentido, é necessário compreender como a produção do conhecimento sobre parâmetros multidimensionais da voz associados à tireoidectomia tem sido abordada nesses periódicos.

Uma das estratégias para estudar como o conhecimento científico sobre um determinado tema é explorado na literatura é o estudo bibliométrico. Este tipo de revisão de literatura traz a possibilidade de analisar o contexto da produção científica publicada a partir de indicadores métricos que representam o desenvolvimento científico da área pesquisada e possibilitam auxiliar a produtividade $\mathrm{e}$ avanço do conhecimento sobre determinado tema. ${ }^{12}$

Sendo assim, o objetivo deste estudo é analisar o perfil da produção científica sobre parâmetros multidimensionais da voz associados à tireoidectomia nos periódicos brasileiros especializados em Fonoaudiologia, no período de 2005 a 2019.

\section{Método}

Trata-se de um estudo de revisão de literatura com abordagem bibliométrica. Trata-se de um estudo documental restrito à análise da literatura científica, portanto, não foi necessário submeter o projeto ao Comitê de Ética e Pesquisa em Seres Humanos.

A coleta foi realizada em janeiro de 2020 .Foram selecionados os periódicos brasileiros especializados em Fonoaudiologia indexados entre 2005 e 2019. Os periódicos foram: Audiology Communication Research (ACR), CoDAS, Distúrbios de Comunicação e Revista CEFAC.

Foram incluídos artigos originais, artigos de revisão, estudos de caso, comunicações breves e resenhas editoriais publicadas no período de interesse e que apresentassem resultados sobre parâmetros perceptivoaauditivos, acústicos e/ou de autoavaliação da voz antes ou após tireoidec- 
tomia. Foram excluídos resumos de dissertações, teses, cartas ao editor e anais de congressos. A busca foi realizada de forma independente por dois pesquisadores na base eletrônica específica de cada periódico, utilizando descritores em português brasileiro disponíveis no DeCS (Descritores em Ciências da Saúde), conforme a seguinte estratégia de cruzamento: disfonia AND (neoplasias da glândula tireoide OR tireoidectomia).

A triagem dos artigos foi realizada primeiramente por meio da leitura dos títulos e resumos e, em seguida, pela leitura completa dos artigos. Como variáveis de análise foram extraídas as seguintes informações: a região em que o estudo foi realizado, instituições que participaram do estudo, quantidade de instituições envolvidas, números de autores que participaram do trabalho, grau e formação acadêmica dos autores (com consulta complementar à Plataforma Lattes), periódico no qual o estudo foi publicado, tipo de artigo, tipo do estudo, tamanho da amostra e tipo de parâmetro vocal avaliado (perceptivoauditivo, acústico e/ou autoavaliação). A análise foi feita de forma descritiva com apresentação de distribuição de frequências absolutas e relativas.

\section{Resultados}

Foram encontrados sete artigos e destes, dois foram eliminados por não cumprirem os critérios de inclusão. Assim, cinco artigos foram selecionados para leitura do texto completo, e para análise bibliométrica, de acordo com o fluxograma apresentado na Figura1.

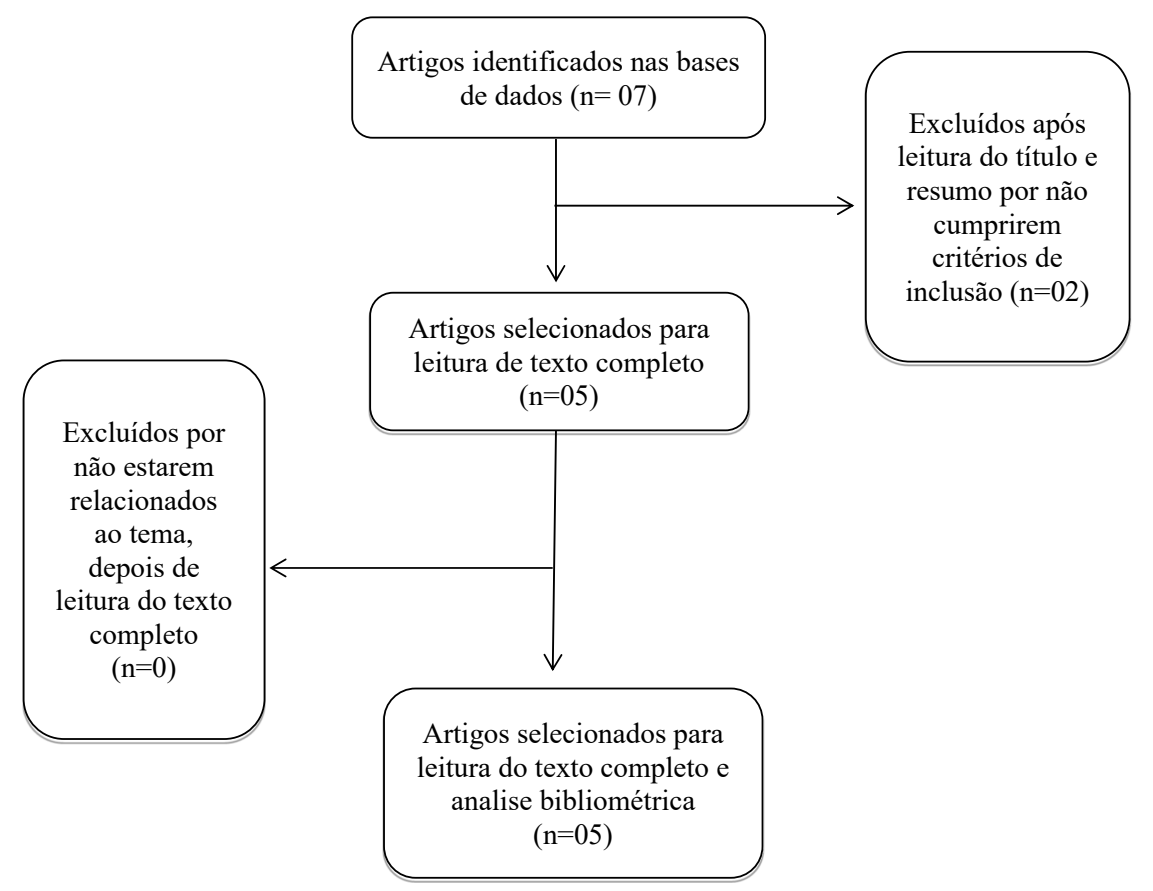

Figura 1. Fluxograma de seleção dos estudos 
As variáveis extraídas dos artigos estão compiladas no Quadro 1. Entre 2005 e 2019, o primeiro artigo sobre o tema foi publicado em 2015 , seguido de um artigo em 2016, dois em 2017 e um em 2019. As regiões Sudeste e Nordeste se destacaram com duas produções publicadas, sendo o outro estudo realizado na região Sul. Quatro trabalhos foram realizados em instituições federais de ensino e dois deles, diferente dos demais, não envolveram parcerias interinstitucionais. O número de autores variou entre três e sete, envolvendo fonoaudiólogos, enfermeiros e médicos. Em apenas um deles o primeiro autor era formado em Enfermagem e não em Fonoaudiologia. $\mathrm{O}$ grau acadêmico do primeiro autor variou entre graduação (dois artigos), mestrado (dois artigos) e doutorado (um artigo).
Dois artigos foram publicados na Revista CEFAC, um na ACR, um na CoDAS e um na Distúrbios da Comunicação. Entre os cinco artigos incluídos nesta revisão, três foram publicados como estudos originais, um como comunicação breve e um como resenha de um artigo internacional. O tamanho amostral variou entre 32 e 60 indivíduos, mas não foi apresentado cálculo amostral e o processo de amostragem foi por conveniência. As quatro produções tinham como foco a autoavaliação vocal do paciente antes e/ou após tireoidectomia. Três estudos abordaram o impacto da voz na qualidade de vida do paciente, e dois fizeram levantamento de sintomas vocais e de deglutição.

Quadro 1. Variáveis referentes aos estudos que abordaram alterações vocais e tireoidectomia, publicados nos periódicos brasileiros especializados em Fonoaudiologia, entre 2005 até 2019.

\begin{tabular}{|c|c|c|c|c|c|c|c|c|c|c|c|c|}
\hline Autores & Ano & Região & $\begin{array}{l}\text { Instituição da } \\
\text { publicação }\end{array}$ & $\begin{array}{c}\text { Número de } \\
\text { instituiçōes }\end{array}$ & $\begin{array}{c}\text { Números de } \\
\text { autores }\end{array}$ & $\begin{array}{l}\text { Formação } \\
\text { do } 1^{\circ} \text { autor }\end{array}$ & $\begin{array}{c}\text { Grau } \\
\text { acadêmico } \\
\text { do } 1^{\circ} \text { autor }\end{array}$ & \begin{tabular}{|c|}
$\begin{array}{c}\text { Formação } \\
\text { dos } \\
\text { coautores }\end{array}$ \\
\end{tabular} & Revista & $\begin{array}{c}\text { Tipo de } \\
\text { artigo e de } \\
\text { estudo }\end{array}$ & $\begin{array}{l}\text { Tamanho da } \\
\text { amostra e } \\
\text { amostragem }\end{array}$ & $\begin{array}{c}\text { Tipo de } \\
\text { parâmetro } \\
\text { vocal avaliado }\end{array}$ \\
\hline $\begin{array}{l}\text { Nascimento } \\
\text { Junior, Carra- } \\
\text { de Angelis, } \\
\text { Lima }(2015)^{13}\end{array}$ & 2015 & Sudeste & $\begin{array}{c}\text { Instituto de } \\
\text { Gerenciamento em } \\
\text { Deglutição - IGD } \\
\text { São Paulo, SP, } \\
\text { Brasil } \\
\end{array}$ & 3 & 3 & $\begin{array}{l}\text { Fonoaudió- } \\
\text { logo }\end{array}$ & Mestrado & $\begin{array}{l}\text { Fonoau- } \\
\text { diologia } \\
\text { Medicina }\end{array}$ & CEFAC & $\begin{array}{l}\text { Artigo } \\
\text { original, } \\
\text { estudo } \\
\text { do tipo } \\
\text { transversal }\end{array}$ & $\begin{array}{c}\mathrm{n}=32 \\
\text { Amostragem } \\
\text { por } \\
\text { conveniência }\end{array}$ & $\begin{array}{l}\text { Autoavaliação - } \\
\text { Qualidade de } \\
\text { vida em voz }\end{array}$ \\
\hline $\begin{array}{l}\text { Koga, Leite, } \\
\text { Ribeiro } \\
(2016)^{14}\end{array}$ & 2016 & Sul & $\begin{array}{c}\text { Hospital das } \\
\text { Clínicas da } \\
\text { Universidade } \\
\text { Federal do } \\
\text { Paraná - HC-UFPR } \\
\text { - Curitiba (PR), } \\
\text { Brasil. } \\
\text { B }\end{array}$ & 3 & 3 & Enfermeira & Mestrado & $\begin{array}{l}\text { Fonoau- } \\
\text { diologia }\end{array}$ & CEFAC & $\begin{array}{c}\text { Comunicação } \\
\text { o breve, } \\
\text { estudo } \\
\text { do tipo } \\
\text { transversal }\end{array}$ & $\begin{array}{c}\mathrm{n}=60 \\
\text { Amostragem } \\
\text { por } \\
\text { conveniência }\end{array}$ & $\begin{array}{l}\text { Autoavaliação - } \\
\text { Qualidade de } \\
\text { vida em voz }\end{array}$ \\
\hline $\begin{array}{l}\text { Guimarães et } \\
\text { al. }(2017)^{15}\end{array}$ & 2017 & Sudeste & $\begin{array}{l}\text { Universidade } \\
\text { Federal do Espirito } \\
\text { Santo, Vitória, ES, } \\
\text { Brasil. }\end{array}$ & 1 & 6 & $\begin{array}{l}\text { Fonoaudió- } \\
\text { loga }\end{array}$ & Doutorado & $\begin{array}{l}\text { Fonoau- } \\
\text { diologia }\end{array}$ & $\begin{array}{l}\text { Distúrbios da } \\
\text { comunicaçãoo }\end{array}$ & Resenha & Não se aplica & Não se aplica \\
\hline $\begin{array}{l}\text { Araujo, Lopes, } \\
\text { Silva, Perrusi, } \\
\text { Farias, } \\
\text { Azevedo } \\
(2017)^{10} \\
\end{array}$ & 2017 & Nordeste & $\begin{array}{c}\text { Universidade } \\
\text { Federal da Paraíba } \\
\text { - UFPB - João } \\
\text { Pessoa (PB), } \\
\text { Brasil. }\end{array}$ & 4 & 6 & $\begin{array}{l}\text { Fonoaudió- } \\
\text { loga }\end{array}$ & Graduação & $\begin{array}{l}\text { Fonoau- } \\
\text { diologia } \\
\text { Medicina }\end{array}$ & CoDAS & $\begin{array}{l}\text { Artigo } \\
\text { original, } \\
\text { estudo } \\
\text { do tipo } \\
\text { transversal }\end{array}$ & $\begin{array}{c}\mathrm{n}=12 \\
\text { Amostragem } \\
\text { por } \\
\text { conveniência }\end{array}$ & $\begin{array}{l}\text { Autoavaliação - } \\
\text { Sintomas de voz } \\
\text { e deglutição }\end{array}$ \\
\hline $\begin{array}{l}\text { Cruz et al. } \\
(2019)^{11}\end{array}$ & 2019 & Nordeste & $\begin{array}{c}\text { Universidade } \\
\text { Federal da Paraíba } \\
\text { - UFPB - João } \\
\text { Pessoa (PB), Brasil }\end{array}$ & 1 & 7 & $\begin{array}{l}\text { Fonoaudió- } \\
\text { loga }\end{array}$ & Graduação & $\begin{array}{l}\text { Fonoau- } \\
\text { diologia }\end{array}$ & ACR & $\begin{array}{l}\text { Artigo } \\
\text { original, } \\
\text { estudo } \\
\text { do tipo } \\
\text { transversal }\end{array}$ & $\begin{array}{c}\mathrm{n}=51 \\
\text { Amostragem } \\
\text { por } \\
\text { conveniência }\end{array}$ & $\begin{array}{l}\text { Autoavaliação - } \\
\text { Sintomas de voz } \\
\text { e deglutição }\end{array}$ \\
\hline
\end{tabular}

\section{Discussão}

Esta revisão bibliométrica observou que entre 2005 e 2019 foram poucas as publicações sobre a associação entre aspectos vocais e tireoidectomia nos periódicos brasileiros especializados em Fonoaudiologia. Considerando o período examinado, o primeiro estudo sobre o tema nesses periódicos foi publicado em 2015, ou seja, houve uma latência de pelo menos 10 anos sem que os periódicos da área de Fonoaudiologia apresentassem alguma produção sobre alterações vocais e tireoidectomia. Ademais, os artigos publicados restringiram seu desfecho a aspectos de autoavaliação funcional ou da qualidade de vida.

Os resultados apontam que o tema ganhou atenção dos periódicos da área de Fonoaudiologia apenas recentemente, provavelmente em função do maior interesse dos pesquisadores da área de Voz no assunto. O investimento nessas pesquisas pode estar relacionado também ao crescimento do número de tireoidectomias nos últimos anos o que implica em mais pacientes com queixas vocais e torna necessária a ampliação das investigações científicas que ofereçam mais suporte à clínica fonoaudiológica. 
Também deve ser ponderado que no período contemplado nesta revisão alguns pesquisadores brasileiros que exploraram o tema podem ter publicado os resultados de seus estudos em periódicos de áreas afins ou periódicos internacionais de mais alto impacto, provavelmente no intuito de expandir o reconhecimento ou visibilidade das suas pesquisas e até mesmo por critérios de exigência dos órgãos que regulam o desenvolvimento da ciência no Brasil. ${ }^{16}$ Supõe-se que a maior receptividade dos periódicos nacionais especializados em Fonoaudiologia à pesquisas acerca do tema, e o crescente avanço na qualidade desses periódicos, poderá mudar esse cenário no futuro e estimular a publicação em periódicos nacionais.

Considerando os resultados desta revisão, percebe-se que há muito a se avançar quanto à abordagem dos aspectos vocais associados à tireoidectomia nos periódicos da área de Fonoaudiologia. Aprimorar os desenhos de estudo para além dos transversais e diversificar o desfecho principal das pesquisas em relação aos parâmetros vocais investigados são perspectivas relevantes nesse processo de avanço. Além disso, são necessários estudos com nível de evidência mais elevado, com amostras mais representativas e oriundas de um processo de amostragem mais robusto.

Sugere-se, portanto, que os pesquisadores da área se apropriem cada vez mais de questões metodológicas e que possam praticar parcerias interinstitucionais no intuito de facilitar a execução de novas pesquisas, já que são notórias as barreiras que o campo da pesquisa encontra no Brasil, especialmente em relação a fomentos que incentivem a concretização de projetos. A execução de projetos de pesquisa de qualidade possui, na sua maioria, um custo elevado, além de requerer tempo e dedicação dos pesquisadores que, além disso, não conseguem se dedicar exclusivamente aos projetos por receberem bolsas incompatíveis com a demanda requisitada. ${ }^{17}$ Dessa forma, compreende-se, em parte, que a totalidade de artigos encontrados tenham um desenho de estudo mais barato, pois são transversais e utilizam, em sua maioria, questionários como materiais de coleta.

Já a diversidade de instituições envolvidas, número de coautores e diferentes formações acadêmicas encontradas nos artigos incluídos nesta revisão apontam um caminho em direção ao fortalecimento das redes de colaboração. Como a pesquisa brasileira tradicionalmente concentra-se nas universidades, em especial nas instituições federais de ensino superior, é natural que esse movimento cresça a partir de grupos vinculados a esses locais. Isso pôde ser percebido pelo maior número de artigos oriundos de grupos de pesquisa de universidades federais, distribuídos pelas regiões Sul, Nordeste e Sudeste. Esperava-se mais expressividade da região Sudeste, pois existem questões históricas de desenvolvimento centralizado das instituições de ensino superior nessa região, que até hoje concentra um relevante polo de universidades e faculdades. ${ }^{18}$ Entretanto, a região Nordeste apareceu com mesmo número de publicações, um indício de mudança de paradigma nesse sentido, provavelmente relacionado ao aumento do número de cursos de graduação e pós-graduação em Fonoaudiologia nessa região nas duas últimas décadas.

Já era esperado que a maioria dos autores fossem fonoaudiólogos, já que a área de voz consiste em uma das especialidades reconhecidas pelo conselho de classe da profissão. Contudo, a integração da Medicina e Enfermagem sinaliza que o tema alcança interesses multiprofissionais, o que amplifica as possibilidades de avanços nesse contexto. Além disso, a construção de um modelo de produção de conhecimento coletivo e colaborativo, no qual a parceria entre academia e sociedade (empresas, entidades públicas, organizações do terceiro setor, etc.) exerce um papel mais ativo e direto na produção do conhecimento parece ser uma alternativa para superar algumas adversidades no âmbito da pesquisa ${ }^{19,20}$.

Os resultados desta revisão bibliométrica sinalizaram que a baixa produtividade encontrada limita as bases científicas que futuros pesquisadores e profissionais interessados pelo tema necessitam ter para aumentar a qualidade das pesquisas e a eficiência nos serviços oferecidos. Sugere-se que outros estudos de revisão bibliométrica ampliem o escopo de investigação deste estudo e analisem qual o padrão de produção sobre o assunto nos periódicos nacionais e internacionais de forma geral.

\section{Conclusão}

Esta revisão bibliométrica apontou que a associação entre aspectos vocais e tireoidectomia foi um tema pouco abordado nos periódicos brasileiros da área de Fonoaudiologia entre 2005 e 2019. O perfil da produção foi de publicações realizadas com parcerias interinstitucionais, predominante- 
mente em universidades federais localizadas nas regiões Sudeste e Nordeste. O primeiro autor tinha formação em Fonoaudiologia na maioria dos casos, com grau acadêmico que variou da graduação ao doutorado. Os coautores incluíram fonoaudiólogos e médicos em quantidade que oscilou entre três e sete. Exceto por uma resenha, os outros estudos foram transversais com amostras que variaram entre 12 e 60 participantes e, em todos, o desfecho principal teve foco na autoavaliação vocal.

\section{Referências}

1. Ritchie JE, Balasubramanian SP. Anatomy of the pituitary, thyroid, parathyroid and adrenal glands. Surgery. 2014; 32(10): 499-503.

2. Robinson KA, Middleton WD. Ultrasonographic evaluation of the thyroid. Ultrasound clin. 2014; 9(3): 325-37.

3. Nam IC, Park YH. Pharyngolaryngeal symptoms associated with thyroid disease. CurrOpinOtolaryngol Head NeckSurg. 2017; 25(6): 469-74.

4. Kane EG, Shore S. Thyroidectomy. Surgery. 2014; 32(10): 543-7.

5. Iyomasa RM, Tagliarini JV, Rodrigues SA, Tavares ELM, Martins RHG. Laryngeal and vocal alterations after thyroidectomy. RevBrasOtorrinolaringol. 2019; 85(1): 3-10.

6. Park YM, Oh KH, Cho JG, Baek SK, Kwon SY, Jung KY, et al. Changes in voice- and swallowing-related symptoms after thyroidectomy: one-year follow-up study. Ann OtolRhinolLaryngol. 2018; 127(3): 171-7.

7. Yu WHV, Wu CW. Speech therapy after thyroidectomy. GlandSurg. 2017; 6(5): 501-09.

8. Ryu J, Ryu YM, Jung Y, Kim S, Lee YJ, Lee E, et al. Extent of thyroidectomy affects vocal and throat functions: A prospective observational study of lobectomy versus total thyroidectomy. Surgery (United States). 2013; 154(3): 611-20.
9. Silva ICM, Netto IP, Vartanian JG, Kowalski LP, Angelis EC. Prevalence of up peraerodigestive symptoms in patients who underwent thyroidectomy with and without the use of intra operative laryngeal nerve monitoring. Thyroid. 2012; 22(8): 814-9.

10. Araújo LF, Lopes LW, Silva POC, Perrusi VJF, Farias VLL, Azevedo EHMA. Sintomas sensoriais em pacientes submetidos à tireoidectomia. CoDAS. 2017; 29(3)1-6.

11. Cruz JS, Lopes LW, Alves GAS, Rodrigues DSB, Xavier DS, Costa BI et al. Combined frequency of patient-reported swallowing and voice complaints before thyroidectomy. Audiology - Communication Research. 2019, 24, e2180.

12. Araújo RF, Alvarenga L. A bibliometria na pesquisa científica da pós-graduação brasileira de 1987 a 2007. Encontros BibliRev eletrônica Biblioteconomia e ciência da informação [Internet]. 2011; 16(31): 51-70.

13. Nascimento Junior JR, Carrara-de Angelis E, Lima ENP. Qualidade de vida relacionada à voz e à deglutição, em curto prazo, em pacientes submetidos à radioiodoterapia por carcinoma diferenciado de tireoide. Rev CEFAC. 2015; 17(2): 396-408.

14. KogaMRV, Leite APD, Ribeiro VV. Qualidade de vida em voz de pacientes no pré-operatório de tireoidectomia. Rev CEFAC. 2016; 18(5): 1035-41.

15. Guimaraes MF, Messa TB, Zordan LQ, Azevedo EHM, Bracet MA, Queiroz JAN. Mudança Temporal na Qualidade Vocal após Tireoidectomia. Distúrb comun. 2017; 29(4): 809-11.

16. Rodrigues LKV, Pernambuco L. Produção científica sobre disfagia orofaríngea em idosos nos periódicos brasileiros: uma análise bibliométrica. Distúrb. comun. 2017; 29(3): 529-38.

17. Corbucci PR. Desafios da educação superior e desenvolvimento no Brasil. Texto Discussão. 2007; 1287: 1-33.

18. Franco AP. Ensino Superior no Brasil: cenário, avanços e contradições. J Polít Educ. 2008; 2(4): 53-63.

19. Gazda E, Quandt CO. Colaboração interinstitucional em pesquisa no Brasil: tendências em artigos na área de gestão da inovação. RAE eletrônica. 2010; 9(2).

20. Martin BR. The changing social contract for science and the evolution of the university. In: Geuna A e outros. (Eds) Science and Innovation: Rethinking the Rationales for Funding and Governance. Cheltenham: Edward Elgar; 2003; 7-29. 